Research article Open Access

\title{
GATA3 protein as a MUC1 transcriptional regulator in breast cancer cells
}

\author{
Martín C Abba ${ }^{1,2}$, María I Nunez¹, Andrea G Colussi², María V Croce ${ }^{2}$, Amada Segal-Eiras ${ }^{2}$ and C \\ Marcelo Aldaz ${ }^{1}$
}

\begin{abstract}
1Department of Carcinogenesis, The University of Texas MD Anderson Cancer Center, Science Park - Research Division, PO Box 389 , Smithville, TX 78957, USA

${ }^{2}$ Centro de Investigaciones Inmunológicas Básicas y Aplicadas (CINIBA), Facultad de Ciencias Médicas, Universidad Nacional de La Plata, Calle 60 y 120 , La Plata, CP:1900, Argentina
\end{abstract}

Corresponding author: Martín C Abba, mabba777@hotmail.com

Received: 1 May 2006 Revisions requested: 16 Jun 2006 Revisions received: 22 Jul 2006 Accepted: 1 Nov 2006 Published: 1 Nov 2006

Breast Cancer Research 2006, 8:R64 (doi:10.1186/bcr1617)

This article is online at: http://breast-cancer-research.com/content/8/6/R64

(c) 2006 Abba et al.; licensee BioMed Central Ltd.

This is an open access article distributed under the terms of the Creative Commons Attribution License (http://creativecommons.org/licenses/by/2.0), which permits unrestricted use, distribution, and reproduction in any medium, provided the original work is properly cited.

\begin{abstract}
Introduction Recent studies have demonstrated that members of the GATA-binding protein (GATA) family (GATA4 and GATA5) might have pivotal roles in the transcriptional upregulation of mucin genes (MUC2, MUC3 and MUC4) in gastrointestinal epithelium. The zinc-finger GATA3 transcription factor has been reported to be involved in the growth control and differentiation of breast epithelial cells. In SAGE (serial analysis of gene expression) studies we observed an intriguing significant correlation between GATA3 and MUC1 mRNA expression in breast carcinomas. We therefore designed the present study to elucidate whether MUC1 expression is regulated by GATA3 in breast cancer cells.
\end{abstract}

Methods Promoter sequence analysis of the MUC1 gene identified six GATA cis consensus elements in the 5 ' flanking region (GATA1, GATA3 and four GATA-like sequences). Chromatin immunoprecipitation and electrophoretic mobilityshift assays were employed to study the presence of a functional GATA3-binding site. GATA3 and MUC1 expression was analyzed in vitro with a GATA3 knockdown assay. Furthermore, expression of GATA3 and MUC1 genes was analyzed by realtime RT-PCR and immunohistochemistry on breast cancerspecific tissue microarrays.

Results We confirmed the presence of a functional GATA3binding site on the MUC1 promoter region in the MCF7 cell line. We determined that GATA3 knockdown assays led to a decrease in MUC1 protein expression in MCF7 and T47D cells. In addition, we detected a statistically significant correlation in expression between GATA3 and MUC1 genes at the mRNA and protein levels both in normal breast epithelium and in breast carcinomas ( $p=0.01$ ). GATA3 expression was also highly associated with estrogen receptor and progesterone receptor status $(p=0.0001)$ and tumor grade $(p=0.004)$ in breast carcinomas.

Conclusion Our study provides evidence indicating that GATA3 is probably a mediator for the transcriptional upregulation of MUC1 expression in some breast cancers.

\section{Introduction}

GATA3 (GATA-binding protein 3) belongs to a family of transcription factors (GATA1 to GATA6) that bind with high affinity to the consensus sequence $(A / T) G A T A(A / G)$ and share a steroid-hormone-receptor superfamily C4 zinc-finger DNAbinding motif [1]. GATA factors are classified into two subfamilies on the basis of structural features and expression pat- terns. The expression of GATA1, GATA2, and GATA3 has been detected predominantly in hematopoietic cells, whereas GATA4, GATA5, and GATA6 are expressed mainly in the cardiovascular system and in endodermal-derived tissues including liver, lung, pancreas, and intestine [2]. The function of GATA factors is modulated by their interaction with other tran-

$\mathrm{bp}=$ base pairs; ChIP = chromatin immunoprecipitation; DAB = diaminobenzidine; DTT = dithiothreitol; ELISA = enzyme-linked immunosorbent assay; EMSA = electrophoretic mobility-shift assay; ER = estrogen receptor; ESR1 = gene encoding estrogen receptor $\alpha$; GATA3 = GATA-binding protein $3 ; \mathrm{HRP}=$ horseradish peroxidase; IDC = invasive ductal carcinoma; $M U C 1=$ mucin $1 ; \mathrm{PR}=$ progesterone receptor; RT-PCR $=$ reverse-transcriptase-mediated polymerase chain reaction; $\mathrm{SAGE}=$ serial analysis of gene expression; TMA $=$ tissue microarray. 
scription factors, transcriptional coactivators and co-repressors.

In genome-wide expression profile studies from our laboratory we observed that the expression of GATA3 is highly correlated with estrogen receptor- $\alpha(E R \alpha)$ status in breast carcinomas [3] similar results were reported by others [4-9]. Parikh and colleagues (2005) suggested that GATA3 expression might be associated with responsiveness to hormone therapy in breast cancer patients [10]. Furthermore, the expression of GATA3 has been shown to correlate with specific breast cancer phenotypes, defined as luminal type A, carrying an improved disease-free survival and overall survival when compared with tumors that do not express GATA3 [11]. It has been reported that GATA3 may be involved in growth control and differentiation in breast epithelial cells mediating the transcriptional activation of several genes such as those encoding cytokeratins 5, 6 and 17, and trefoil factors 1 and 3 [12]. Recent evidence indicates that the proteins GATA4, GATA5, and GATA6 may be important in the upregulation of mucin expression (MUC2, MUC3, and MUC4) and trefoil factor genes (TFF1 and TFF2), events that are in turn associated with gastrointestinal epithelial cell differentiation [13-15].

The MUC1 glycoprotein is a member of the mucin family of proteins, expressed mostly on the apical membrane of various glandular epithelia such as in luminal breast epithelial cells [16]. The association of MUC1 overexpression with loss of cell polarity has been observed in breast carcinomas. Abnormal MUC1 expression leads to a loss of cell-cell and cellextracellular-matrix adhesion [17]. It was further determined that this increase in MUC1 expression is due mainly to transcriptional regulatory events [18].

The 5'-regulatory region of the human MUC1 gene was analyzed previously [19-21]. Several consensus binding sites for transcription factors were observed in this promoter region, such as those for the SP1, STAT1, STAT3, NF- $\kappa B, M Z F 1$ and DbpA proteins, all of which may be involved in the transcriptional regulation of MUC1 [18,20,22]. However, the factors determining MUC1 tissue-specific expression remain largely unknown, as do the mechanisms causing MUC1 overexpression in tumors.

Global gene expression studies pointed to a significant correlation in the overexpression of GATA3 and MUC1 genes commonly observed in breast cancer. Interestingly, we also observed the presence of multiple putative GATA-binding sites throughout the MUC1 promoter. Thus, the aim of this study was to evaluate the role of GATA3 as a putative transcriptional regulator of MUC1 in breast cancer.

\section{Materials and methods}

\section{Serial analysis of gene expression database mining}

To perform a comparative analysis of the GATA family members expressed in breast tissue, we analyzed 47 breast SAGE (serial analysis of gene expression) libraries: 4 normal breast epithelium, 8 ductal carcinoma in situ (DCIS), 33 invasive ductal carcinomas (IDCs), and the MCF7 and ZR75 breast cancer cell lines. To this end, we combined 29 breast cancer SAGE libraries generated by us at a resolution of 100,000 tags per library (Aldaz Laboratory) with 18 SAGE libraries (generated at the Polyak Laboratory, Dana-Farber Cancer Institute, Boston, MA, USA) downloaded from the Cancer Genome Anatomy Project - SAGE Genie database [23]. SAGE data management and tag-to-gene matching for GATA1 (GCCTCCAGAG), GATA2 (GACAGTTGTT), GATA3 (AAGGATGCCA), GATA4 (TCTCTCCCCT), GATA5 (TCCTGGCATA), GATA6 (GAGAAGATCA), ESR1 (AGCAGGTGCC), and MUC1 (CCTGGGAAGT) were performed with a suite of web-based SAGE library annotation tools developed by us [24]. To enable the visualization and illustration of our analyses, we used the TIGR MultiExperiment Viewer (MeV 2.2) software (The Institute for Genomic Research, Rockville, MD, USA). This tool was employed for normalization and average clustering of the SAGE data. Spearman's test was employed for a correlation analysis of transcripts. The CGAP-dbEST and Oncomine databases [25] were employed for collection and visualization of gene expression profiles of the previously mentioned transcripts from publicly available breast cancer ESTs and microarray data sets (Additional file 1).

\section{Real-time RT-PCR expression analysis in breast carcinomas}

For validation studies, total RNA was isolated from an independent set of 36 snap-frozen breast carcinomas (stages I and II: $13 \mathrm{ER} \alpha$-negative tumors and $23 \mathrm{ER} \alpha$-positive tumors) using TRIzol reagent (Invitrogen, San Francisco, CA, USA). Template cDNAs were synthesized with the SuperScript ${ }^{\mathrm{TM}}$ First-strand Synthesis System (Invitrogen). GATA3 and MUC1 primers and probes were obtained from Applied Biosystems (TaqMan Assays-on-Demand ${ }^{\mathrm{TM}}$ Gene Expression Products; Foster City, CA, USA). All the PCR reactions were performed with the TaqMan PCR Core Reagents kit and the ABI Prism ${ }^{\circledR} 7700$ Sequence Detection System (Applied Biosystems). Experiments were performed in duplicate for each data point and 18S rRNA was used as control. Results are expressed as means \pm 2 SEM based on $\log _{2}$ transformation of normalized real-time RT-PCR values of the assayed genes.

\section{Tissue microarray and immunostaining analysis}

We used breast-specific tissue microarrays (TMAs) from two sources (Fox Chase Cancer Center, Philadelphia, and Cooperative Breast Cancer Tissue Resource, NCl-CBCTR) for GATA3 and MUC1 protein expression profiling. We analyzed a total of 263 cases (38 normal tissues (19 DCIS and 206 
IDCs). Information on ER and progesterone receptor (PR) status was available for all IDC cases. Before immunostaining, endogenous peroxidase activity was blocked with $3 \% \mathrm{H}_{2} \mathrm{O}_{2}$ in water for 10 minutes. Heat-induced epitope retrieve was performed with $1.0 \mathrm{mM}$ EDTA buffer $\mathrm{pH} 8.0$ for 10 minutes in a microwave oven followed by cooling for 20 minutes. To block non-specific antibody binding, the slides were incubated with $10 \%$ goat serum in PBS for 30 minutes. Primary monoclonal anti-GATA3 (HG3-31: SC-268; Santa Cruz Biotechnology, Santa Cruz, CA, USA) and anti-MUC1 (VU4H5: SC-7313; Santa Cruz Biotechnology) antibodies were used at 1:50 and 1:100 dilutions respectively. Antibody detection was performed using diaminobenzidine (DAB). Staining intensity was determined by means of a Chromavision Automated Cellular Imaging System $\left(\mathrm{ACIS}^{\circledR}\right)$ with the generic DAB software application, as described previously [26]. This software determines brown intensity regardless of the area covered by the positive cells.

Multivariate analysis was performed by principal components analysis (PCA). Variables were codified and transformed as follows: negative staining ( 0 ) and positive staining (1) for ER, PR, GATA3 and MUC1 expression; normal tissue (0), DCIS (1) and IDC (2) for histology; lymph node-negative (0) and lymph node-positive (1) status; and low (1), moderate (2) and high (3) tumor grade. To enable visualization of the factorial analysis, we employed a three-dimensional representation of component plot in rotated space. The basic significance level was fixed at $p<0.05$ and all data were analyzed with SPSS ${ }^{\circledR}$ statistical software (SPSS Inc., Chicago, IL, USA).

\section{Cell culture, western blot and immunocytochemistry analyses}

The estrogen-dependent breast cancer cell lines MCF7 and T47D were grown in RPMI-1640 medium (Gibco, Gaithersburg, MD, USA) supplemented with 10\% (v/v) FBS (Bioser, Buenos Aires, Argentina), $10 \mathrm{U} / \mathrm{ml}$ penicillin $\mathrm{G}$, and $10 \mu \mathrm{g} / \mathrm{ml}$ streptomycin.

Trypan blue staining was used to assess cell viability. Cells ( 3 $\left.\times 10^{6}\right)$ were lysed with $1 \mathrm{ml}$ of RIPA buffer $(50 \mathrm{mM}$ Tris $\mathrm{pH} 7.5$, $150 \mathrm{mM} \mathrm{NaCl}, 0.5 \%$ sodium deoxycholate, $1 \%$ Triton $\mathrm{X}-100$, $0.1 \%$ SDS) containing protease inhibitor. For western blotting, $50 \mu \mathrm{l}$ of cell extract was separated by $10 \%$ SDS-PAGE and transferred to nitrocellulose membrane (Protran ${ }^{\circledR}$; BioScience, Dassel, Germany). Immunodetection was performed with a GATA3 mouse monoclonal antibody (HG3-31) at 1:300 dilution, followed by incubation with horseradish peroxidase (HRP)-conjugated secondary antibody (anti-mouse-HRP; Sigma, St Louis, MO, USA) at 1:1000 dilution. Immunostaining was performed with DAB as substrate.

MCF7 cells were also grown on coverslips; these preparations were washed twice with $1 \times$ PBS and fixed with $10 \%$ formaldehyde in $1 \times$ PBS. Immunodetection of GATA3 was assessed with HG3-31 monoclonal antibody. Primary antibodies were detected with biotin-conjugated goat anti-mouse lgG, followed by incubation with streptavidin-conjugated HRP and using DAB as a substrate. The cells grown on coverslips were counterstained with hematoxylin and examined by light microscopy.

\section{MUC1 promoter sequence analysis and chromatin immunoprecipitation}

The complete sequence of the human MUC1 promoter region was obtained from GenBank (accession no. NM 002456). We used the DNAMAN software (Version 4.15; Lynnon BioSoft, Vaudreuil-Dorion, Quebec, Canada) to identify putative GATA-binding sites (A/T-GATA-A/G) in this regulatory sequence.

MCF7 breast cancer cells were grown to $90 \%$ confluence; culture medium was then removed. Cells were washed and fixed with $1 \%$ formaldehyde in $1 \times$ PBS for 10 minutes at room temperature. Fixed cells were washed twice with $1 \times$ PBS, scraped off and incubated in $1 \mathrm{ml}$ of lysis buffer $A(20 \mathrm{mM}$ HEPES pH 7.9, 25\% glycerol, $420 \mathrm{mM} \mathrm{NaCl}, 1.5 \mathrm{mM} \mathrm{MgCl}_{2}$, $0.2 \mathrm{mM}$ EDTA, $1 \mathrm{mM}$ phenylmethylsulphonyl fluoride), followed by a second incubation in $300 \mu$ l of lysis buffer $B(50$ $\mathrm{mM}$ Tris- $\mathrm{HCl} \mathrm{pH}$ 8.0, $1 \mathrm{mM}$ EDTA, $150 \mathrm{mM} \mathrm{NaCl}, 1 \%$ SDS, $2 \%$ Triton X-100). The cell lysate was then sonicated with a Branson Sonifier 450 (30-second pulses at $40 \%$ output) and subjected to immunoprecipitation with mouse monoclonal antibody HG3-31 against GATA3, at $4^{\circ} \mathrm{C}$ for 1 hour; $50 \mu \mathrm{l}$ of Protein A-Sepharose (1:1 slurry) was added to the reaction and incubated at $4^{\circ} \mathrm{C}$ for 4 hours. The unbound proteins were removed by washing the Protein A-Sepharose with Triton buffer (50 mM Tris- $\mathrm{HCl} \mathrm{pH}$ 8.0, $1 \mathrm{mM}$ EDTA, $150 \mathrm{mM} \mathrm{NaCl}$, $0.1 \%$ Triton $\mathrm{X}-100)$ and $1 \times$ PBS. The antigen-antibody complex was eluted with SDS- $\mathrm{NaCl}$-dithiothreitol (DTT) buffer (62.5 mM Tris-HCl pH 6.8, $200 \mathrm{mM} \mathrm{NaCl}, 2 \%$ SDS, $10 \mathrm{mM}$ DTT). The eluted protein-DNA complex was incubated overnight at $68^{\circ} \mathrm{C}$. DNA was isolated with the phenol/chloroform extraction and ethanol precipitation protocol.

The PCR reactions were performed with $15 \mathrm{ng}$ of DNA, 2.5 $\mathrm{mM} \mathrm{MgCl} 2$, each dNTP at $200 \mu \mathrm{M}, 25$ pmol of muc1p1 forward primer (5'-tagaagggtggggctattcc-3'), $25 \mathrm{pmol}$ of muc1p1 reverse primer (5'-taggtcgaggtcctgtacag-3', flanking the GATA1-binding site), 25 pmol of muc1p2 forward primer (5'-tttggctgatttggggatgc-3'), 25 pmol of muc1p2 reverse primer (5'-aatattgcactcgtcccgtc-3', flanking the GATA3-binding site), and 1.25 units of Taq DNA polymerase (Promega, Madison, WI, USA), in PCR buffer (20 mM Tris-HCl pH 8.4, $50 \mathrm{mM} \mathrm{KCl}$ ) in a final volume of $50 \mu \mathrm{l}$. The reactions were cycled as follows: 1 cycle of $94^{\circ} \mathrm{C}$ for 2 minutes and 25 cycles of $94^{\circ} \mathrm{C}$ for 1 minute, $56^{\circ} \mathrm{C}$ for 1 minute, and $72^{\circ} \mathrm{C}$ for 1 minute. HLA-DQ $\alpha 1$ amplicon (242 bp) was used as control (exon sequence without a GATA-binding site) [27]. Separation and detection of the amplified fragments were performed 
by electrophoresis on a $6 \%$ minigel (19:1 polyacrylamide:bisacrylamide) and staining with silver.

\section{Electrophoretic mobility-shift assay}

Nuclear protein extract from the MCF7 cell line was prepared as described [28]. The following double-stranded DNA probes (27 bp) were used: the wild-type GATA consensus oligonucleotide (5'-cacttgataacagaaagtgataactct-3') as positive control [4]; the MUC1 promoter sequence encoding the putative GATA3-binding site (5'-ggcggatctttgatagactggagtgtc-3'); and a double GATA3-binding site mutated variant (with transitions $\mathrm{G} \rightarrow \mathrm{C}$ and $\mathrm{A} \rightarrow \mathrm{T}$ : 5'-ggcggatcttcttagactggagtgtc-3'). A non-isotopic electrophoretic mobility-shift assay (EMSA) was performed with $5 \mu \mathrm{g}$ of nuclear cell extracts and $50 \mathrm{ng}$ of the probe, $1 \mu \mathrm{g}$ of salmon testes DNA (Sigma-Aldrich, St Louis, $\mathrm{MO}, \mathrm{USA})$ in a $5 \times$ binding reaction buffer $(100 \mathrm{mM}$ Hepes $\mathrm{pH}$ $7.9,250 \mathrm{mM} \mathrm{KCl}, 2.5 \mathrm{mM}$ DTT, $0.25 \mathrm{mM}$ EDTA, $5 \mathrm{mM} \mathrm{MgCl}_{2}$, $25 \%$ glycerol). After 20 minutes the samples were separated on a $10 \%$ polyacrylamide (39:1 polyacrylamide:bisacrylamide) at a constant temperature of $4^{\circ} \mathrm{C}$ with $1 \times$ Tris-borate-EDTA (45 mA for 4.5 hours). Gels were stained with a previously described silver staining technique [27]. Similarly, for the supershift assay, $1 \mu \mathrm{g}$ of specific antibody (anti-GATA3) or control antibody (anti-ER $\beta$ ) was incubated for 20 minutes in the MCF7 nuclear protein extract before the addition of probes. Samples were size separated by electrophoresis in a $10 \%$ polyacrylamide minigel (39:1 polyacrylamide:bisacrylamide) at room temperature with $0.5 \times$ Tris-borate-EDTA.

\section{GATA3 anti-sense phosphorothioate oligonucleotide assay}

Anti-sense oligodeoxynucleotides were synthesized that matched the translational start region of GATA3 $\left(5^{\prime}\right.$-cgccgtcacctccatggcctc-3') [29]. The GATA3 anti-sense oligodeoxynucleotide used was synthesized on a phosphorothioate backbone to improve resistance to endonucleases (IDT, Coralville, IA, USA). MCF7 and T47D cells were plated on $50 \mathrm{~mm}$ cultured dishes at $50 \%$ confluence in Opti-MEM ${ }^{\circledR}$ I Reduced Serum Medium (Invitrogen) and transiently transfected with 3 $\mu \mathrm{g}$ of GATA3 anti-sense mixed with Lipofectamine in accordance with the manufacturer's protocol (Invitrogen). Cells were maintained at $37^{\circ} \mathrm{C}$ and harvested at two time points after transfection (48 and 72 hours). Cells were lysed in RIPA buffer, and total protein concentration was estimated by Lowry's method. Equal amounts of protein were used for the western blot analyses. GATA3 and MUC1 immunodetection were assessed with HG3-31 and CT2 (epitope corresponding to the carboxy terminus of MUC1) monoclonal antibodies, respectively. Primary antibodies were detected with biotinconjugated goat anti-mouse IgG (for HG3-31) or biotin-SPconjugated goat anti-Armenian hamster IgG (for CT2) (Jackson ImmunoResearch), followed by incubation with streptavidin-conjugated HRP and using DAB as a substrate. SDSPAGE with silver staining was employed for the detection of total protein loaded.
In addition, we analyzed the expression of MUC1 protein and its response to GATA3 anti-sense by an ELISA assay with MCF7 cell culture. In brief, MCF7 cells were cultured on a 96well microtitre plate and treated with $0.05 \mu \mathrm{g}$ of GATA3 antisense (48 hours), and then blocked with 1\% BSA. MUC1 immunodetection was performed with HMFG1 monoclonal antibody. The bound primary antibody was detected with peroxidase-conjugated goat anti-mouse IgG. Color was developed with ABTS (2,2'-Azinobis (3-ethylbenzthiazoline-6sulfonic acid)) substrate solution and absorbance was measured at $405 \mathrm{~nm}$ with a microplate reader (SLT Spectra, SLT Labinstruments, Salzburg, Austria). MUC1 expression was expressed as mean optical density with a $95 \%$ confidence interval. Statistically significant difference was estimated by $t$ test $(p<0.05)$.

\section{Results \\ GATA transcription factors family members expressed in breast epithelial cells}

Interestingly, among the GATA family members GATA3 was by far the most frequently expressed GATA transcription factor in breast cancer. Specifically, GATA3 was detected as being expressed in $90 \%$ of the breast cancer cases (42 out of 47) according to SAGE database analyses. In contrast, GATA family members GATA1, GATA2, GATA4, GATA5, and GATA6 were detected as expressed in only $13 \%$ (6 out of 47 ) of the SAGE libraries (Figure 1). Similarly, expressed sequence tag database and DNA microarray analyses showed that the GATA3 gene is expressed mainly in breast carcinomas compared with other GATA genes (Additional file 1). The MCF7 breast cancer cell line displayed the highest GATA3 expression level observed. We detected a significant positive correlation between ESR1 (the gene encoding ER $\alpha$ ) and GATA3 tags $(r=0.702 ; p=0.0001)$, between ESR1 and MUC1 $(r=0.46 ; p=0.001)$, and between GATA3 and $\operatorname{MUC1}(r=0.45 ; p=0.002)$ (Figure 1).

\section{GATA3 and MUC1 real-time RT-PCR analysis in breast carcinomas}

To validate the relationship of GATA3 and MUC1 in breast cancer epithelial cell further, we performed an mRNA expression analysis of 36 primary breast carcinomas by means of real-time RT-PCR. These data showed a significant positive correlation between the mRNA levels of both markers $(r=0.6$; $p<0.0001$ ) (Figure 2a). Furthermore, mRNA expression of GATA3 and MUC1 correlated positively with breast carcinomas ER $\alpha$ status $(r=0.492, p=0.004 ; r=0.608, p=0.001$, respectively) (Figure $2 \mathrm{~b}, \mathrm{c}$ ).

\section{GATA3 and MUC1 tissue-microarray analysis}

PCA of 263 breast tissue samples (Figure 3a; Additional file 2) identified statistically significant positive correlations between GATA3 expression and ER/PR status $\left(\tau_{\mathrm{b}}=0.53 ; p=\right.$ 0.001 ) (Figure $3 a, b$ ). It is interesting to note that GATA3 and MUC1 protein expression are positively correlated among nor- 


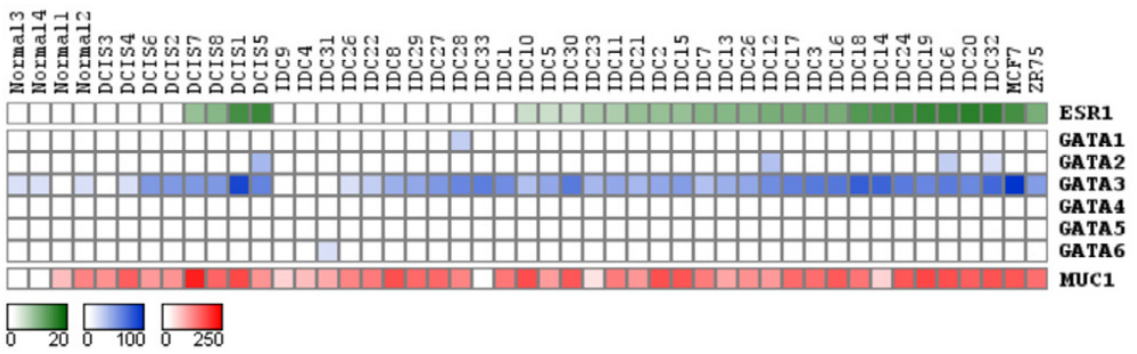

Digital northern analysis based on SAGE data of GATA family members, ESR1 and MUC1 genes in 47 breast SAGE libraries. The color scale at the bottom represents expression level based on numbers of transcripts (tags) per library. SAGE, serial analysis of gene expression.

mal breast samples as well as in breast carcinomas $\left(\tau_{b}=0.44\right.$; $p=0.01$ ). Additionally, MUC1 expression was correlated with lymph node-positive status in breast carcinomas $\left(\tau_{b}=0.46 ; p\right.$ $=0.006$ ). We detected a strong correlation between the lack of GATA3 expression and higher histological tumor grade (poorly differentiated) demonstrated by multivariate $\left(\tau_{\mathrm{b}}=\right.$ $0.49 ; p=0.002)$ and univariate $(p=0.004)$ analyses (Figure $3 a, c$ ). Whereas $54.3 \%$ (19 out of 35 ) of IDC grade I and $45 \%$ (40 out of 89 ) of IDC grade II tumors were GATA3-positive, only $27 \%$ (18 out of 67 ) of IDC grade III tumors were GATA3positive $(p=0.013)$.

Overall, these studies showed a consistently statistically significant correlation between GATA3 and MUC1 expression at the mRNA and protein levels by SAGE profiling $(p=0.002)$, real-time RT-PCR $(p<0.0001)$, and TMA $(p=0.01)$ in breast carcinomas.

\section{Identification of GATA cis-elements within MUC1 promoter}

Analysis of the MUC1 promoter sequence indicated the presence of six putative GATA binding sites at $-446 /-451$ (GATA1), -1444/-1449 (GATA-like), -1572/-1577 (GATAlike), -2398/-2393 (GATA3), -2475/-2470 (GATA-like), and 2602/-2607 (GATA-like) (Figure 4). These sites encode the conserved consensus sequence required for the binding of GATA factors. To determine whether GATA3 is capable of binding the $-2398 /-2393$ sequence in vivo, we performed a chromatin immunoprecipitation (ChIP) assay with nuclear extracts obtained from the MCF7 breast cancer cell line. In brief, protein-DNA complexes were crosslinked in vivo, sonicated and then immunoprecipitated with anti-GATA3 monoclonal antibodies. DNA samples were obtained from three fractions: input, non-specific eluted, and immunoprecipitated. These DNA samples were subjected to PCR with two sets of primers that flanked the predicted GATA3-binding site (2398/-2393) and the putative GATA1-binding site (-446/451 ) in the MUC1 promoter region.

In addition, we employed a set of primers spanning the second exon of the HLA-DQA1 gene (242 bp), used as negative control because there are no GATA-binding motifs in its sequence. The amplified product corresponding to the GATA1-binding site (186 bp) was detected from input DNA and non-specific GATA3-eluted DNA fractions (Figure 5a). However, this GATA1-related amplified product was not detected from the anti-GATA3-immunoprecipitated DNA fraction (Figure $5 \mathrm{a}$ ), indicating that the GATA3 protein does not recognize the $-446 /-451$ site from the MUC1 promoter region.

Figure 2
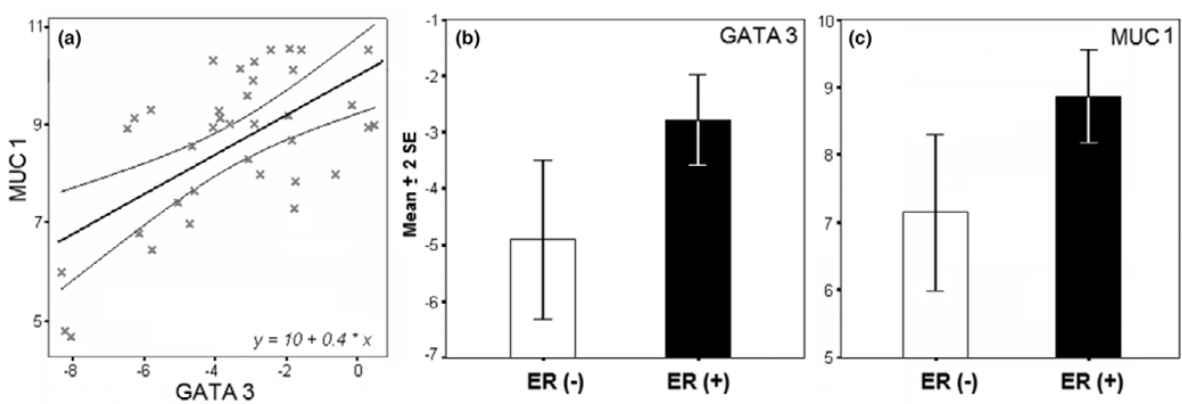

GATA3 and MUC1 real-time RT-PCR analysis in 36 invasive breast carcinomas. (a) Linear regression analysis of GATA3 and MUC1 mRNA expression levels in invasive ductal carcinoma (IDC) with 95\% mean prediction interval $(r=0.6 ; p<0.0001)$. (b,c) GATA3 ( $p=0.008)(b)$ and MUC1 ( $p$ $=0.009$ ) (c) mRNA expression of IDC in association with ER $\alpha$ status. Results are real-time RT-PCR values of the assayed gene relative to $18 \mathrm{~S}$ rRNA used as normalizing control and are shown as means \pm 2 SEM based on a $\log _{2}$ transformation. 
Figure 3
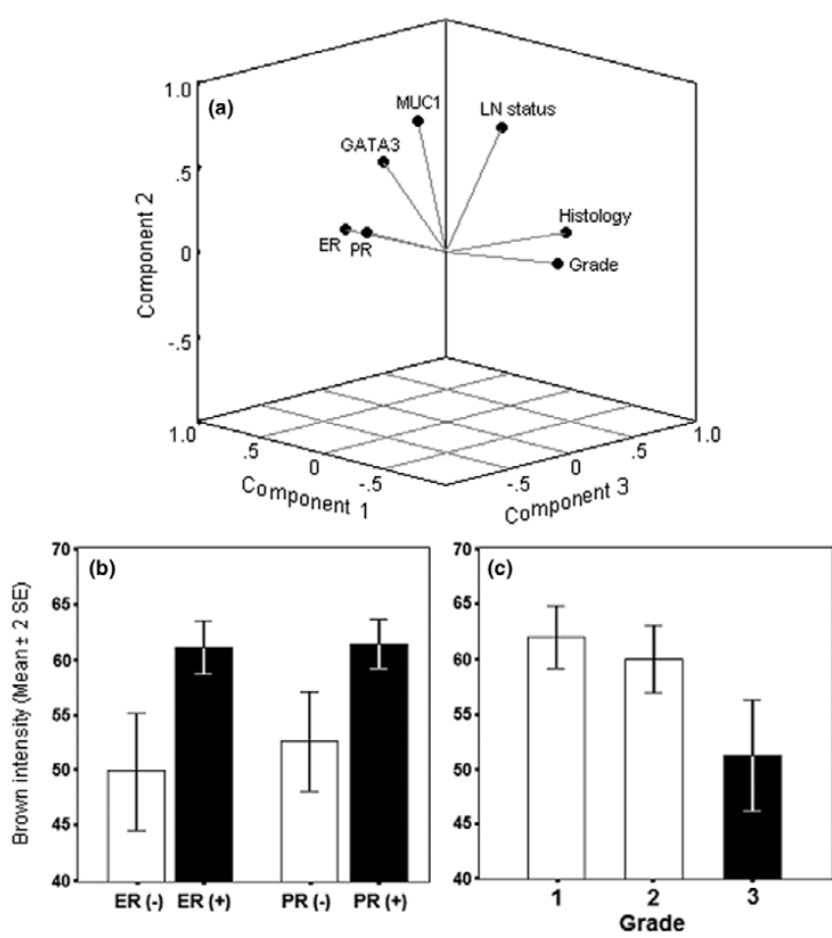

Tissue microarray analysis of 263 breast normal and cancer tissue samples. (a) Component plot in rotated space showing factors positively correlated, namely estrogen receptor/progesterone receptor (ER/PR; $p$ $=0.0001)$, ER-PR/GATA3 $(p=0.001)$, and MUC1/lymph node status $(p=0.006)$, GATA3/MUC1 $(p=0.01)$; and factors negatively correlated, namely ER-PR/tumor grade $(p=0.0001)$ and GATA3/tumor grade $(p=0.002)$. $(\mathbf{b}, \mathbf{c})$ Univariate analysis showing highly statistical association between GATA3 immunostaining and ER and PR status ( $p$ $=0.0001)(\mathrm{b})$ and statistical decrease trend in GATA3 immunostaining and the tumor grade of invasive component $(p=0.004)$ (c). Results are based on the brown intensity of immunostaining as described in the Materials and methods section, and are shown as means \pm 2 SEM.

Identical results were obtained for the HLA-DQA1-amplified products (negative control), demonstrating the specificity of the ChIP assay (Figure 5b). In contrast, the amplified product spanning the GATA3-binding site (292 bp in size) was detected in the input and anti-GATA3-immunoprecipitated DNA fractions (Figure 5c). These amplified products were detected only in the second immunoprecipitated DNA fraction (reChIP assay; Figure 5e). Furthermore, we employed an EMSA approach to validate the ChIP findings. Nuclear extract retarded the mobility of the GATA3 sequence-specific probe (positive control) and the probe encoding the putative GATA3binding site (5'-ggcggatctttgatagactggagtgtc-3') found in the wild-type MUC1 promoter region (-2398/-2393) (Figure 6). In contrast, electromobility was not affected when a point mutant of the aforementioned MUC1 probe (T/CTTA/G instead of the wild-type T/GATA/G) was employed, indicating that no protein binding occurred (Figure 6). The presence of GATA3 in the retarded probe-protein complex was confirmed by supershift analysis (Figure 6b). The addition of anti-GATA3 antibody to the binding reaction showed a further decrease in the mobility of the probe-protein complex. Addition of the control antibody (anti-ER $\beta$ ) had no effect.

Overall, these data indicate that endogenous GATA3 protein molecules from the MCF7 breast cancer cell line were able to associate with the putative GATA3-binding site of MUC1 promoter sequence, as demonstrated with in vivo and in vitro assays.

\section{GATA3 anti-sense assay}

To investigate the effect of GATA3 on MUC1 gene expression, a GATA3 knock-down assay was performed with specific anti-sense oligonucleotides in MCF7 and T47D breast cancer cell lines. Cells were treated with optimal concentrations of GATA3 anti-sense oligonucleotides $(3 \mu \mathrm{g} / \mathrm{ml})$ and examined 48 and 72 hours after transfection. Immunoblotting analyses of the cells treated with MCF7 and T47D showed that GATA3 translation was blocked by the anti-sense oligonucleotide at both time points analyzed (namely at 48 and 72 hours after transfection; Figure 7a). Treated cells showed a significant decrease in MUC1 expression (Figure 7). Total protein staining showed that these results were not due to differences in protein loading. The same result was obtained in a separate experiment with a MCF7-cell ELISA assay with a different monoclonal antibody (HMFG1) against MUC1 (Figure 7b).

\section{Discussion}

Transcriptome profile analyses demonstrated that GATA3 expression is strongly correlated with $E R \alpha$ status in breast cancer [3-9]. Recently we performed a cross-platform comparison between SAGE and DNA microarray profiles showing that the ESR1, GATA3, and MUC1 genes are very tightly coexpressed in breast carcinomas (Figure 1) [3]. To validate the relationship of GATA3 and MUC1 in breast tissues further, we performed mRNA expression analyses of 36 invasive breast carcinomas by real-time RT-PCR, and protein expression analysis in 263 breast tissue samples by means of immunohistochemistry on TMAs. Both analyses showed a statistical positive correlation between GATA3 and MUC1 expression at the mRNA and protein levels ( $p<0.01$; Figures $2 \mathrm{a}$ and $3 \mathrm{a}$ ). Interestingly, we also detected a positive correlation between GATA3 and MUC1 protein expression when both markers were evaluated in normal breast tissue as well $(p=0.001)$.

Two recent studies from gastrointestinal epithelial cells indicated that GATA family members (GATA4 and GATA5) may have pivotal roles in the transcriptional upregulation of three mucin genes: MUC2, MUC3, and MUC4 [14,15]. These data led us to infer that GATA3 might have a role in the transcriptional upregulation of MUC1 in breast epithelium.

To determine whether GATA3 protein was actively bound to the predicted consensus MUC1 promoter sequence (at - 
(a)

(b)

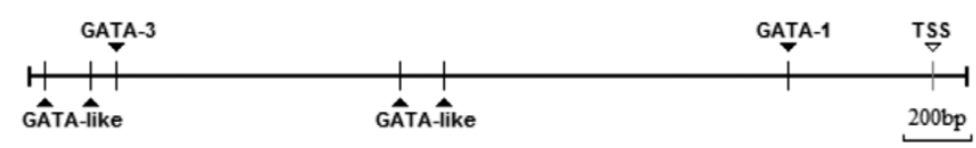

-2651 TIGTTTTCTAAGCCAGTGCAACTGCTTATTTIAGGGATTTICCGGCATAGGGTGGGAAGTGGAAGGATCGGCGAGTAG

-2571 AAGAGAAACCTGGGAGGTTGAAGTAGGGATCTAGGGAAGTTTGGCTGATTTGGGGATGCGGGTGGGGGAGTGCTG

-2491 GATGGAGTIAAGTGAAGATAGGGTGCCTGAGGGAGGATGCCCGAAGTCCTCCCAGACCCACTTACTCACGGTGGCAGC

-2411 GCGACACTCCAGICTATCAAAGATCCGCCGGGATGGAGAGCCAGGAGGCGGGGGCIGCCCCIGAGGIAGCGGGGAGGCCG

-2331 GGGGGCCGGGGGCGGACGGGACGAGTGCAATATTGGCGGGGGAAAAACAACACTGCACCGCGTCCGTCCCTCCCGCC

-2251 CGCCCGGGCCCGGATCCCGCTCCCCACCGCCTGAAGCCGGCCCGACCCGCAACCCGGGCCGCTGGGGAGTTGGGTTCACC

-2171 TIGGAGGCCAGAGAGACTIGGCGCCCGGAAGCAAAGGGAIGGCAAGGGGAGGGGGGAGGGAGAACGGGAGTITGCGGA

-2091 GTCCAGAAGGCCGCTITCCGACGCCCGGGCGTTECGCGCGCTIGCTCTITAAGTACTCAGACTGCGCGGCGCGAGCCGTC

-2011 CGCATGGTGACGCGTGTCCCAGCAACCGAACTGAATGGCTGTTGCTTGGCAATECCGGGAGTTGAGGTTIGGGGCCGCCC

-1931 ACCTAGCTACTCGTGTITICTCCGGCCTGCGAGTIGGGGGGCTCCCGCCTCCCCGGCCCGCTCCTGGGCGCGCTGACGTC

-1851 AGATGTCCCCACCCCGCCCAGCGCCTGCCCCAAGGGTCTCGCCGCACACAAAGCTCGGCCTCGGGCGCCGGCGCGCGGGC

-1771 GAGAGCGGTGTCTCTCGCCTGCTGATCTGATGCGCTCCAATCCCGTGCCTCGCCGAAGTGTTTTIAAAGTGTTCTTTCC

-1691 AACCTGTGTCTITGGGCCTGAGAACIGTTITCTGAATACAGGCGGAACTGCTICCGICGGCCTAGAGGCACGCTGCGACT

-1611 GCGGCACCCAAGTTCCACGTGCTGCCGCGCCCTGGGATAGCTTCCTCCCCTCGTGCACTGCTGCCGCACACACCTCTTGG

-1531 CIGTCGCGCATTACGCACCTCACGIGTGCTITTECCCCCCGCTACGTGCCTACCTGTCCCCAATACCACICTGCTCCCCA

-1451 AAGGATAGITCTGIGICCGTAAATCCCATTCTGICACCCACCTACTCICTGCCCCCCCCITIITIGITITGAGACGGAG

-1371 CITTGCTCTGICGCCCAGGCTGGAGTGCAATGGCGCGATCTCGGCICACTGCAACCTCCGCCTCCCGGGITCAAGCGATT

-1291 CTCCTGCCTCAGCCTCCTGAGTAGCTGGGGTACAGCECCCGCACCACGCTCGGCTAATTTTTGTAGTTTTTAGTAGAG

-1211 ACGAGGITTCACCATCTIGGCCAGGCTGGICTTEAACCCCTEACCTIGTGATCCACTCGCCTCGGCCITCCAAAGTGTIG

-1131 GGATTACGGGCGIGACGACCGTGCCACGCATCTGCCTCTTAAGTACATAACGGCCCACACAGAACGTGTCCAACTCCCCC

-1051 GCCCACGTTCCAACGTCCICTCCCACATACCTCGGTGCCCCTICCACATACCTCAGGACCCCACCCGCTIAGCTCCATIT

-971 CCTCCAGACGCCACCACCACGCGTCCCGGAGTGCCCCCTCCTAAAGCTCCCAGCCGTCCACCATGCTGTGCGTTCCTCCC

-891 TCCCTGGCCACGGCAGTGACCCTICTCTCCCGGCCCCTGCTICCCTCTCGCGGGCTCTGCTECCTCACTIAGGCAGCGCT

-811 GCCCTIACTCCTCTCCGCCCGGTCCAGCGGCCCCTCAGCTTCGGCGCCCAGCCCCGCAAGCCTCCCGGTGACCACTAGA

-731 GGGCGGAGAGCTCCIGGCCAGIGGIGGAGAGIGGCAAGGAAGGACCCIAGGGITCATCGGAGCCAGGITIACTCCCT

-651 IAAGTGGAAATITCTICCCCCACICCICCTIGGCITICICCAAGGAGGGAACCCAGGCTGCIGGAAAGTCCGGCTGGGGC

-571 GGGGACTGTGGGTICAGGGAGAACGGGTGTGGAACGGACAGGGAGCGTTAGAAGGGTGGGCTATTCCGGGAAGT

-491 GIGGGGGAGGGAGCCCAAAACTAGCACCTAGTCCACTCATTATCCAGCCTCTTATTTCTCGGCCGCTCTGCTICAGTG

-411 GACCCGGGCAGGCCGGGGAAGTGAGTGGGAGACCTAGGGGTGGGCTTCCCGACCTIGCTGIACAGACCTCGACCTAGC

-331 TGGCTTTGTTCCCCATCCCCACGTIAGTTGTTGCCCTGAGGCTAAAACTAGAGCCCAGGGGCCCAAGTTCCAGACTGCC

-251 CTCCCCCCTCCCCCGGAGCCAGGCAGTGGTTGGTGAAAGGGGAGGCCAGCTGGAGAACAAACGGGTAGTCAGGGGGTT

-171 GAGCGITAGAGCCCITGIACCCIACCCAGGATGGITGGGCAGGAGGAGGAGAGGIAGGAGGTAGGGAGGGGCGGG

-91 GITITGICACCTGICACCTGCTCGCTGIGCCTAGGGCGGCGGGCGGGAGIGGGGGGACCGGTATAAAGCGTAGGCGC

-11 CTGTGCCCGCTCCACCTCTCAAGCAGCCAGGCCTGCCTGAATCTGTTCTGCCCCCTCCCCACCCAITTCACCACCACCATG $\underset{\text { TSS }}{\longrightarrow}$

MUC1 promoter sequence. (a) GATA cis-elements map identified in MUC1 promoter region. (b) DNA sequence showing the TATA box (white box), the transcriptional start site +1 (TSS arrow) and the open reading frame (ORF arrow). Gray boxes indicate putative binding sites for GATA transcription factors showing the following: a GATA1 sequence (-448), a GATA3 sequence (-2395) and four GATA-like sequences $(-1446,-1574,-2472$, and -2604). Known regulatory elements are underlined (Sp1, STAT1/3, E-MUC1).

2398/-2393 from the transcriptional start site (TSS)) in vivo and in vitro, we performed ChIP and gel shift analyses in breast cancer cells. The ChIP assay in MCF7 demonstrated the existence of a functional GATA3 binding site within the MUC1 promoter (Figure 5). The EMSA studies further validated ChIP data (Figure 6). Having found that GATA3 transcription factor efficiently recognizes a sequence motif in the distal portion of the MUC1 promoter, we proceeded to analyze the effects of GATA3 suppression on MUC1 protein expression in transiently transfected MCF7 cells. GATA3 knockdown assays led to a significant decrease in MUC1 protein expression in MCF7 breast cancer cells (Figure 7), strongly suggesting an involvement of GATA3 in the modulation of MUC1 expression.

The MUC1 promoter sequence has been analyzed previously for its ability to direct the expression of a reporter gene [1921]. The construct (including $2.9 \mathrm{~kb}$ of MUC1 5'-flanking region) showed high expression levels in ZR75 cells but surprisingly low levels in MCF7 and T47D cell lines. This study showed that tissue-specific expression of a reporter gene could be obtained with only $743 \mathrm{bp}$ of 5 ' sequences of the MUC1 gene, using the ZR75 cell line [19]. However, the binding of GATA3 to the promoter regions of target genes gener- 
Figure 5

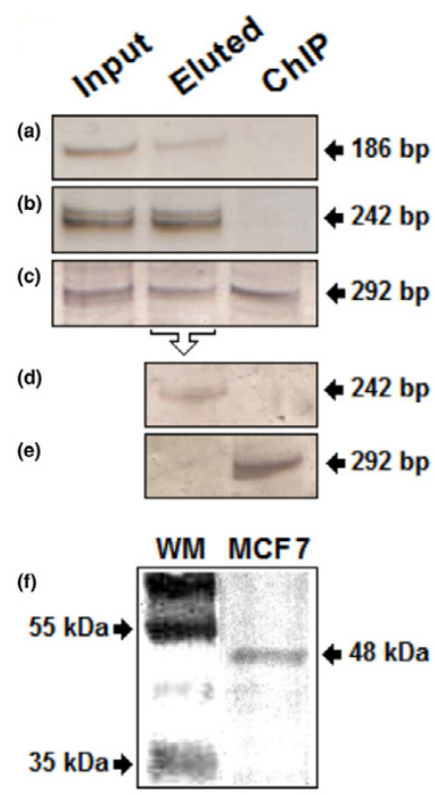

Chromatin immunoprecipitation assay of GATA3 in MCF7 breast cancer cells. Chromatin complexes were crosslinked in vivo with formaldehyde (see the Materials and methods section). The GATA3-associated DNA fragments were immunoprecipitated (IP) with mouse monoclonal antibody against GATA3 (HG3-31). DNA samples were isolated before IP (lane labeled 'input'), after elution the unbound proteins from the Protein A-Sepharose batch (lane labeled 'eluted') and after specific IP (lane labeled 'ChIP', for chromatin immunoprecipitation). (a-c) PCR was performed with (a) flanking primers amplifying a GATA1 binding site on the MUC1 promoter (186 bp; see Figure 2), (b) flanking primers for amplification of exonic sequences of the HLA-DQA1 gene (242 bp; negative control), and (c) flanking primers for amplification of the GATA3-binding site on the MUC1 promoter (292 bp) as shown in Figure 2. (d-e) Second ChIP (reChIP) assay using the first eluted DNAprotein complex as input sample. (f) Western blot analysis of GATA3 protein expression: a sample of MCF7 protein extract was run on a $10 \%$ SDS-PAGE gel and assayed for GATA3 expression with the monoclonal antibody used in ChIP assays (HG3-31). The expected wildtype band of $48 \mathrm{kDa}$ was immunodetected. Lane WM shows an SDS7B prestained SDS-molecular mass standard (Sigma-Aldrich).

ate an open chromatin configuration that increases the accessibility to other main transcriptional regulators (perhaps STAT) in the vicinity of the TSS [30,31]. These data, together with the fact that GATA3 knock-down expression did not immediately abolish MUC1 expression, suggest that GATA3 is partly required to drive $M U C 1$ expression. The finding that GATA3 and MUC1 expression were correlated in SAGE, realtime RT-PCR and TMA immunohistochemistry analyses shows statistical significance. It is also apparently a set of breast carcinomas in which there was no direct correlation between levels of GATA3 and MUC1 expression. This suggests that GATA3 may contribute to MUC1 upregulation in a subset of breast cancer cells (namely ER $\alpha$-positive breast carcinoma).

The role of GATA3 as a transcriptional upregulator of MUC1 could be directly via a cis-acting interaction and/or indirectly
Figure 6

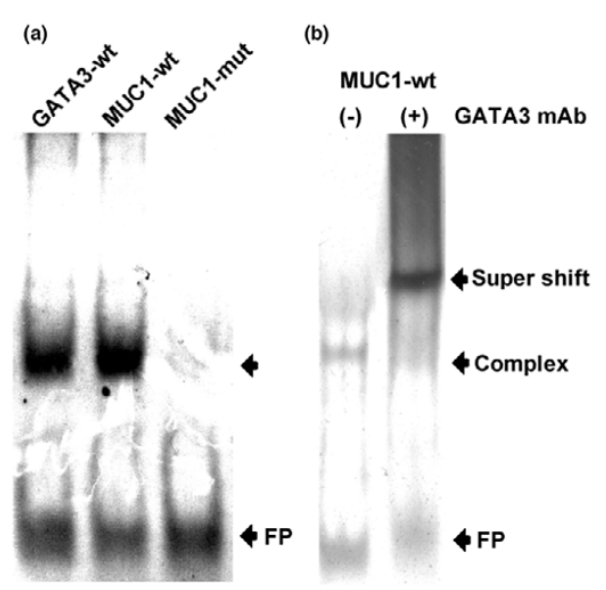

Electrophoretic mobility-shift assay employing cell nuclear extract from MCF7. (a) Gel shift analyses were performed with double-stranded oligonucleotides (27 bp) as indicated: positive control probe containing the GATA3-binding element (GATA3-wt), a mimetic wild-type MUC1 probe containing the predictive GATA3-binding site (MUC1-wt), and a probe sequence with predictive GATA3-binding site mutations (MUC1mut). (b) The presence of GATA3 was confirmed by supershift assay. Nuclear extract prepared from MCF7 cells was preincubated with GATA3 antibody, and then incubated with the mimetic wild-type MUC1 probe containing the GATA3-binding site (MUC1-wt). Arrows to the right indicate the position of the free probe (FP), the probe-nuclearprotein complex (Complex), and the specifically retarded proteinGATA3-antibody-probe complex (Supershift).

via cross-talk between GATA3 and MUC1 co-activators such as ER $\alpha / S P 1$, STAT or FOG (friend-of-GATA cofactors). The transcriptional regulatory event, operating after the binding of GATA3 to the MUC1 promoter, remains to be determined. The MUC1 promoter contains several cis-elements specific for transcription in lymphoid cells such as STAT, MZF1, XBP1, MYB and GATA [21]. The involvement of GATA and STAT proteins in cytokine signaling is well documented in the hematopoietic and lymphoid systems [30]. Interestingly, a GATASTAT synergy has been recently suggested as a general event that could account for cell-specific effects of transcriptional regulation [31]. The presence of these elements, which can upmodulate gene expression in lymphoid cells, are in accord with the observation that the MUC1 gene is expressed in activated $T$ cells [32].

Alternatively, several putative estrogen and progesterone response elements (ERE/PRE) have been identified in the human MUC1 promoter by sequence analysis [33]. Zhou and colleagues [34] demonstrated that ER does not directly regulate the Muc1 promoter sequence in mice. It is possible that the MUC1 gene is transcriptionally regulated by non-EREmediated mechanisms, for example those involving $\mathrm{ER} \alpha$ binding to a co-activator such as AP1 or SP1 [35]. It is interesting to note that two SP1-binding sites have been characterized as important MUC1 transcriptional activators in epithelial cells $[19,20]$. Moreover, there are studies demonstrating protein- 
Figure 7

(a)

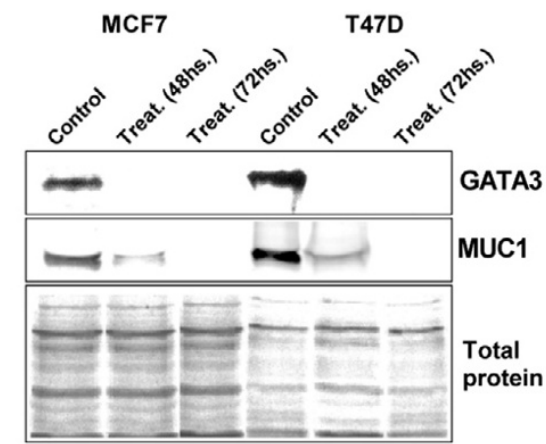

(b)

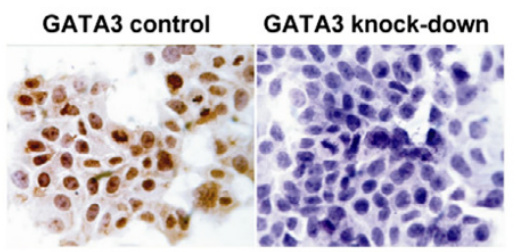

(c)

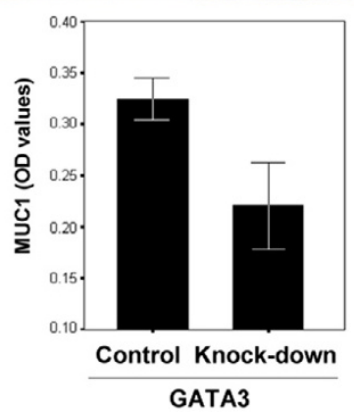

GATA3 knock-down analysis in MCF7 and T47D breast cancer cell lines. (a) Western blot analysis of GATA3 and MUC1 proteins in nontreated (control) and GATA3 anti-sense treated cells ( 48 and 72 hours) in both breast cancer cell lines. SDS-PAGE silver protein staining showed equal amounts of total protein loaded. (b) MCF7 immunocytochemistry of GATA3 anti-sense transfected cells showing no immunoreactivity to the anti-GATA3 antibody, and matching control cells show GATA3-positive nuclear localization. (c) Quantification of MUC1 protein expression by MCF7 culture ELISA assay. GATA3 anti-sense treated cells ( 48 hours) showed a statistically significant decrease in MUC1 expression compared with matched control cells $(p=0.001)$. Each experiment was performed in triplicate.

protein interaction between ER $\alpha$ and GATA1 in erythroid cells $[36,37]$. ER $\alpha$ binds to the GATA1 protein at two sites, one of which is a zinc-finger domain that is highly conserved among GATA family members [36].

Therefore, a possible way of explaining ESR1, GATA3, and MUC1 co-expression could very probably involve the formation of a complex between the ER $\alpha$ and GATA3 proteins that results in the upmodulation of MUC1 gene expression.

It has been shown that high expression of GATA3 correlates with low tumor grade by in silico analysis of cDNA microarray data [12]. As is known, tumor grade is a good measure of breast cancer progression and differentiation status. In this study we have demonstrated a strong correlation between loss of GATA3 protein expression and more advanced stages of breast cancer progression; that is, high-grade less differentiated breast carcinomas expressed lower levels of GATA3 protein on the basis of immunohistochemical studies ( $p=$ 0.004). Similarly, Mehra and colleagues [38] showed that loss of GATA3 expression is associated with higher histological grade.

Our data agree with previously reported studies showing the role of GATA family members in the development and maintenance of a more differentiated state. Interestingly, there is emerging evidence that membrane-associated mucins (MUC1 and MUC4) contribute to the regulation of differentiation, morphogenesis, and proliferation of breast epithelial cells $[17,39]$. Recent data show that a fragment of the MUC1 cytoplasmic tail can be translocated to the nucleus, driving the transcriptional co-activator status of $\beta$-catenin [40]. This finding sustains the possibility that GATA3 might influence cell differentiation possibly through processes involving the upregulation of MUC1 gene expression.

\section{Conclusion}

Our findings suggest that GATA3 could contribute to the transcriptional upregulation of MUC1 gene expression in some breast carcinomas.

\section{Competing interests}

The authors declare that they have no competing interests.

\section{Authors' contributions}

MCA conceived the study idea and carried out the experimental analyses and the biostatistical/bioinformatics analysis and took part in writing the manuscript. MIN coordinated and performed pathological and tissue microarray immunohistochemical studies. AGC and MVC participated in cell culture/ immunohistochemistry studies and provided practical feedback on aspects of the manuscript. CMA and AS are principal investigators and were involved in the conceptualization, design and writing of the manuscript. All authors read and approved the final manuscript.

\section{Additional files}

The following Additional files are available online:

\section{Additional file 1}

A PDF file showing a cross-platform comparison of GATA family members expressed in breast carcinomas using SAGE, expressed sequence tag and DNA microarray databases.

See http://www.biomedcentral.com/content/ supplementary/bcr1617-S1.pdf 


\section{Additional file 2}

A PDF file showing a principal components analysis of tissue microarray data.

See http://www.biomedcentral.com/content/ supplementary/bcr1617-S2.pdf

\section{Acknowledgements}

The authors gratefully acknowledge Dr Andres JP Klein-Szanto (Fox Chase Cancer Center), who kindly provided breast cancer TMAs; Nancy W Abbey for her outstanding technical assistance, Dr Marina Isla-Larrain for the development of the ELISA assay, and Dr J Carri for help with the photography. This work was supported by $\mathrm{NIH}-\mathrm{NCl}$ Grant 1 U19 CA84978-1A1 (C.M. Aldaz) and center grant ES-07784, FONCYT (PICT N05-06544, BID 1201/OC/AR 1253), CONICET, CICPBA and University of La Plata (Argentina).

\section{References}

1. Merika M, Orkin SH: DNA-binding specificity of GATA family transcription factors. Mol Cell Biol 1993, 13:3999-4010.

2. Patient RK, McGhee JD: The GATA family (vertebrates and invertebrates). Curr Opin Genet Dev 2002, 12:416-422.

3. Abba MC, Hu Y, Sun H, Drake JA, Gaddis S, Baggerly K, Sahin A, Aldaz CM: Gene expression signature of estrogen receptor $\alpha$ status in breast cancer. BMC Genomics 2005, 6:37.

4. Hoch RV, Thompson DA, Baker RJ, Weigel RJ: GATA-3 is expressed in association with estrogen receptor in breast cancer. Int J Cancer 1999, 84:122-128.

5. Perou CM, Sorlie T, Eisen MB, van de Rijn M, Jeffrey SS, Rees CA, Pollack JR, Ross DT, Johnsen H, Akslen LA, et al.: Molecular portraits of human breast tumors. Nature 2000, 406:747-752.

6. Gruvberger S, Ringner M, Chen Y, Panavally S, Saal LH, Borg A, Ferno M, Peterson C, Meltzer PS: Estrogen receptor status in breast cancer is associated with remarkably distinct gene expression patterns. Cancer Res 2001, 61:5979-5984.

7. West M, Blanchette C, Dressman H, Huang E, Ishida S, Spang R, Zuzan H, Olson JA, Marks JR, Nevins JR: Predicting the clinical status of human breast cancer by using gene expression profiles. Proc Natl Acad Sci USA 2001, 98:11462-11467.

8. van't Veer LJ, Dai H, van de Vijver MJ, He YD, Hart AA, Mao M, Peterse HL, van der Kooy K, Marton MJ, Witteveeb AT, et al.: Gene expression profiling predicts clinical outcome of breast cancer. Nature 2002, 415:530-536.

9. Sotiriou C, Neo S, McShane LM, Korn EL, Long PM, Jazaeri A, Martiat $P$, Fox SB, Harris AL, Liu ET: Breast cancer classification and prognosis based on gene expression profiles from a population-base study. Proc Natl Acad Sci USA 2003, 100:10393-10398

10. Parikh P, Palazzo JP, Rose LJ, Daskalakis C, Weigel RJ: GATA-3 expression as a predictor of hormone response in breast cancer. J Am Coll Surg 2005, 200:705-710.

11. Sorlie T, Perou CM, Tibshirani R, Aas T, Geisler S, Johnsen H, Hastie T, Eisen MB, van de Rijn M, Jeffrey SS, et al.: Gene expression patterns of breast carcinomas distinguish tumor subclasses with clinical implications. Proc Natl Acad Sci USA 2001, 98:10869-10874.

12. Usary J, Llaca V, Karaca G, Presswala S, Karaca M, He X, Lang$\operatorname{erod}$ A, Karesen R, Oh DS, Dressler LG, et al.: Mutation of GATA3 in human breast tumors. Oncogene 2004, 23:7669-7678.

13. Al-azzeh ED, Fegert $P$, Blin N, Gott P: Transcription factor GATA6 activates expression of gastroprotective trefoil genes TFF1 and TFF2. Biochim Biophys Acta 2000, 1490:324-332.

14. Ren C, Akiyama Y, Miyake S, Yuasa Y: Transcription factor GATA-5 selectively up-regulates mucin gene expression. J Cancer Res Clin Oncol 2004, 130:245-252.

15. van der Sluis M, Melis MHM, Jonckheere N, Ducourouble MP, Buller HA, Renes I, Einerhand AW, Van Seuningen I: The murine Muc2 mucin gene is transcriptionally regulated by the zinc-fin- ger GATA-4 transcription factor in intestinal cells. Biochem Biophys Res Commun 2004, 325:952-960.

16. Gendler SJ: MUC1, the renaissance molecule. J Mammary Gland Biol Neoplasia 2001, 6:339-353.

17. Hollingsworth MA, Swanson BJ: Mucins in cancer: protection and control of the cell surface. Nat Rev Cancer 2004, 4:45-60.

18. Gaemers IC, Vos HL, Volders HH, van der Valk SW, Hilkens J: A STAT-responsive element in the promoter of Episialin/MUC1 gene is involved in its overexpression in carcinoma cells. $J$ Biol Chem 2001, 276:6191-6199.

19. Kovarik A, Peat N, Wilson D, Gendler SJ, Taylor-Papadimitriou J: Analysis of the tissue-specific promoter of the MUC1 gene. Biol Chem 1993, 268:9917-9926.

20. Kovarik A, Lu PJ, Peat N, Morris J, Taylor-Papadimitriou J: Two GC boxes (Sp1 sites) are involved in regulation of activity of the epithelium-specific MUC1 promoter. J Biol Chem 1996, 271:18140-18147.

21. Zaretsky JZ, Sarid R, Aylon Y, Mittelman LA, Wreschner DH, Keydar I: Analysis of the promoter of the MUC1 gene overexpressed in breast cancer. FEBS Lett 1999, 461:189-195.

22. Shiraga $T$, Winpenny JP, Carter EJ, McCarthy VA, Hollingsworth MA, Harris A: MZF-1 and DbpA interact with DNase I hypersensitive sites that correlate with expression of the human MUC1 mucin gene. Exp Cell Res 2005, 308:41-52.

23. Cancer Genome Anatomy Project - SAGE Genie [http:// cgap.nci.nih.gov/SAGE/]

24. Science Park Research Division - Sage Library Tool [http:// spi.mdacc.tmc.edu/bitools/about/sage lib tool.html]

25. Oncomine - Cancer Profiling Database [http://www.oncom ine.org]

26. Nunez MI, Ludes-Meyers J, Abba MC, Kil H, Abbey NW, Page RE, Sahin A, Klein-Szanto AJ, Aldaz CM: Frequent loss of WWOX expression in breast cancer: correlation with estrogen receptor status. Breast Cancer Res Treat 2005, 89:99-105.

27. Abba MC, Gomez MA, Golijow CD: HLA-DQA1 allele typing by nonisotopic PCR-LIS-SSCP. Braz J Med Biol Res 2001, 34:867-869.

28. Lahiri DK, Ge Y: Electrophoretic mobility shift assay for the detection of specific DNA-protein complex in nuclear extracts from the cultured cells and frozen autopsy human brain tissue. Brain Res Protocols 2000, 5:257-265.

29. Umetani M, Mataki C, Minegishi N, Yamamoto M, Hamakubo T, Kodama T: Function of GATA transcription factors in induction of endothelial vascular cell adhesion molecule-1 by tumor necrosis factor- $\alpha$. Arterioscler Thromb Vasc Biol 2001, 21:917-922.

30. Zhu J, Min B, Hu-Li J, Watson CJ, Grinberg A, Wang Q, Killeen N, Urban JF, Guo L, Paul WE: Conditional deletion of Gata3 shows its essential function in Th1-Th2 responses. Nat Immunol 2004, 5:1157-1165.

31. Wang J, Paradis $\mathrm{P}$, Aries A, Komati $\mathrm{H}$, Lefebvre $\mathrm{C}$, Wang $\mathrm{H}$, Nemer $\mathrm{M}$ : Convergence of protein kinase $\mathrm{C}$ and JAK-STAT signaling on transcription factor GATA-4. Mol Cell Biol 2005, 25:9829-9844.

32. Agrawal B, Krantz M, Parker J, Longenecker BM: Expression of MUC1 mucin on activated human T cells: implications for a role of MUC1 in normal immune regulation. Cancer Res 1998, 58:4079-4081.

33. Brayman M, Thathiah A, Carson DD: MUC1: a multifunctional cell surface component of reproductive tissue epithelia. Reprod Biol Endocrinol 2004, 2:4.

34. Zhou X, DeSouza MM, Julian J, Gendler SJ, Carson DD: Estrogen receptor does not directly regulate the murine Muc-1 promoter. Mol Cell Endocrinol 1998, 143:65-78.

35. Gruber CJ, Gruber DM, Gruber IML, Wieser F, Huber JC: Anatomy of the estrogen response element. Trends Endocrinol Metab 2004, 15:73-78.

36. Blobel GA, Sieff CA, Orkin SH: Ligand-dependent repression of the erythroid transcription factor GATA-1 by the estrogen receptor. Mol Cell Biol 1995, 15:3147-3153.

37. Blobel GA, Orkin SH: Estrogen-induced apoptosis by inhibition of the erythroid transcription factor GATA-1. Mol Cell Biol 1996, 16:1687-1694.

38. Mehra R, Varambally S, Ding L, Shen R, Sabel MS, Ghosh D, Chinnaiyan AM, Kleer CG: Identification of GATA3 as a breast cancer prognostic marker by global gene expression metaanalysis. Cancer Res 2005, 65:11259-11263. 
39. Hudson MJ, Stamp GW, Chaudhary KS, Hewitt R, Stubbs AP, Abel PD, Lalani EN: Human MUC1 mucin: a potent glandular morphogen. J Pathol 2001, 194:373-383.

40. Wen Y, Caffrey TC, Wheelock MJ, Johnson KR, Hollingsworth MA: Nuclear association of the cytoplasmic tail of muc1 and $\beta$-catenin. J Biol Chem 2003, 278:38029-38039. 